\title{
Remarks on asymptotic regularity and fixed points
}

\author{
Jarosław Górnicki®
}

\begin{abstract}
Asymptotic regularity allows to provide simple proofs of Banach's theorem and Kannan's theorem. Using asymptotic regularity and Kannan's type conditions we generalize these results, in particular, the Banach contraction principle (see Theorem 2.6 and Corollary 2.10). Further, we discuss the analogous results for monotone mappings on preordered metric spaces, where a preordered binary relation is weaker than a partial order. Next, we will prove a random version of the presented deterministic fixed-point theorems.
\end{abstract}

Mathematics Subject Classification. Primary 47H10; Secondary 47H07, $47 \mathrm{H} 40,54 \mathrm{H} 25,60 \mathrm{H} 25$.

Keywords. Asymptotic regularity, complete metric space, fixed point, Banach contraction principle, Kannan mapping, monotone mapping, preordered space, measurable space, Polish space, random operator, random variable, random fixed point.

\section{Banach and Kannan theorems}

A sequence $\left\{x_{n}\right\}$ in a metric space $(X, d)$ is asymptotically regular if

$$
d\left(x_{n}, x_{n+1}\right) \rightarrow 0 \text { as } n \rightarrow \infty
$$

The condition (1) does not guarantee a convergence of the sequence $\left\{x_{n}\right\}$. Notice that we cannot deduce, that a subsequence $\left\{x_{n_{k}}\right\}_{k}$ of an asymptotically regular sequence $\left\{x_{n}\right\}$ is also asymptotically regular. For example, consider the sequence $\left\{x_{n}=\sum_{i=1}^{n} \frac{1}{i}, n \geqslant 1\right\}$ in the Euclidean space $\mathbb{R}$.

Let $T: X \rightarrow X$ be a mapping. For a initial point $x_{0} \in X$, define a sequence of iterates $x_{n+1}=T x_{n}=T^{n+1} x_{0}, n=0,1,2, \ldots$, and the resulting sequence $\left\{x_{n}\right\}$ is called the sequence of successive approximations of $T$.

Hillam [28] proved:

Theorem 1.1. Let $T$ be a continuous map of $[0,1]$ into $[0,1]$. The sequence $\left\{x_{n}=T^{n} x\right\}$ of successive approximations of $T$ converges to a fixed point of $T$ if and only if (1) holds. 
Smart [44] showed that this result does not extend beyond onedimensional case:

Example 1.2. There is a continuous mapping $T$ of the closed unit disc in the Euclidean plane such that the origin and points on the unit circle are fixed points and every other point $x$ satisfies $d\left(T^{n} x, T^{n+1} x\right) \rightarrow 0$ but $\left\{T^{n} x\right\}$ is not convergent, see [44] for details.

The following observation is trivial:

Lemma 1.3. If $T$ is a continuous map of $X$ into $X$ and if $d\left(T^{n} x, T^{n+1} x\right) \rightarrow 0$, then any limit point $p$ of the set $\left\{T^{n} x\right\}$ is a fixed point of $T$.

Proof. If $T^{n_{k}} x \rightarrow p \in X$, then

$$
p=\lim _{k \rightarrow \infty} T^{n_{k}} x=\lim _{k \rightarrow \infty} T^{n_{k}+1} x=T\left(\lim _{k \rightarrow \infty} T^{n_{k}} x\right)=T p .
$$

Thus, the continuity of a mapping $T: X \rightarrow X$ and the fact that the sequence of successive approximations $\left\{T^{n} x\right\}$ satisfies (1) does not guarantee the existence of a fixed point. For a guarantee that there is a (unique) fixed point, additional assumptions are needed.

Banach's contraction principle [4] is remarkable in its simplicity, because the contractive condition on the mapping is simple and easy to test, because it requires only a complete metric space for its setting, and because it finds almost canonical applications in the theory of differential and integral equations. In this part, we will give an elementary proof of this result exposing (1), for other proofs see [17, Chapter 2], [18].

Let us recall a few facts.

Definition 1.4. Let $(X, d)$ be a metric space and let $T: X \rightarrow X$ be a mapping. $T$ is called a contraction if there exists a fixed constant $0 \leqslant L<1$ such that

$$
d(T x, T y) \leqslant L \cdot d(x, y) \text { for all } x, y \in X
$$

Each contraction is a continuous mapping.

There are many mappings of this type.

Example 1.5. Let $X=[a, b]$ be with usual metric and $T: X \rightarrow X$ be a continuous mapping such that $T$ is differentiable at every $x \in(a, b)$ such that $\left|T^{\prime}(x)\right| \leqslant L<1$. Then, by the mean value theorem, if $x, y \in X$, there is a point $c$ between $x$ and $y$ such that

$$
|T x-T y|=\left|T^{\prime}(c)\right| \cdot|x-y| \leqslant L \cdot|x-y| .
$$

Here is an elementary proof of Banach's contraction principle.

Theorem 1.6 (Banach contraction principle). Let $(X, d)$ be a complete metric space, then each contraction $T: X \rightarrow X$ has a unique fixed point $p \in X$, and $T^{n} x \rightarrow p$ for each $x \in X$. 
Proof. Choose $x_{0} \in X$ arbitrarily and define a sequence $\left\{x_{n+1}=T x_{n}, n=\right.$ $0,1,2, \ldots\}$ of $T$ based on $x_{0}$. Since $T$ is a contraction

$$
d\left(x_{n+1}, x_{n}\right) \leqslant L \cdot d\left(x_{n}, x_{n-1}\right) \leqslant \cdots \leqslant L^{n} \cdot d\left(T x_{0}, x_{0}\right) \rightarrow 0 \text { as } n \rightarrow \infty .
$$

By triangle rule, for any $n$ and any $k>0$, we have

$$
\begin{aligned}
& d\left(x_{n+k+1}, x_{n+1}\right) \leqslant L \cdot d\left(x_{n+k}, x_{n}\right) \\
& \quad \leqslant L \cdot\left\{d\left(x_{n+k}, x_{n+k+1}\right)+d\left(x_{n+k+1}, x_{n+1}\right)+d\left(x_{n+1}, x_{n}\right)\right\},
\end{aligned}
$$

so by $(3)$

$d\left(x_{n+k+1}, x_{n+1}\right) \leqslant \frac{L}{1-L} \cdot\left\{d\left(x_{n+k}, x_{n+k+1}\right)+d\left(x_{n+1}, x_{n}\right)\right\} \rightarrow 0$ as $n \rightarrow \infty$.

Hence $\left\{x_{n}\right\}$ is a Cauchy sequence in a complete metric space $X$ and there exists $p \in X$ such that $x_{n} \rightarrow p \in X$. Because $T$ is continuous and $x_{n+1}=$ $T x_{n}$, it follows that $p=T p$. Suppose $q$ is another fixed point of $T$. Then

$$
0<d(p, q)=d(T p, T q) \leqslant L \cdot d(p, q)<d(p, q),
$$

a contradiction. Hence $T$ has unique fixed point $p \in X$. Because

$$
d\left(T^{n} x, p\right)=d\left(T^{n} x, T^{n} p\right) \leqslant L^{n} \cdot d(x, p) \rightarrow 0 \text { as } n \rightarrow \infty,
$$

we have $T^{n} x \rightarrow p$ for any $x \in X$.

Remark 1.7. The following trivial fact is noteworthy in that the mapping $T$ is not even assumed to be continuous:

Let $(X, d)$ be a complete metric space and $T: X \rightarrow X$ be a mapping for which $T^{N}$ is contraction for some positive integer $N>1$, then $T$ has a unique fixed point.

Not only contractions guarantee the existence of a unique fixed point and the possibility of its approximation. In 1968, Kannan [32] established the following theorem, see [25].

Theorem 1.8. If $T$ is a map of the complete metric space $(X, d)$ into itself and if there exists $0 \leqslant K<\frac{1}{2}$ satisfying

$$
d(T x, T y) \leqslant K \cdot\{d(x, T x)+d(y, T y)\} \quad \text { for all } x, y \in X,
$$

then $T$ has a unique fixed point $p \in X$, and $T^{n} x \rightarrow p$ for each $x \in X$.

Kannan's theorem is important because Subrahmanyam [46] proved that Kannan's theorem characterizes the metric completeness. That is, a metric space $(X, d)$ is complete if and only if every mapping satisfying (4) on $X$ with constant $K<\frac{1}{2}$ has a fixed point. Contractions do not have this property; Connell [11] gave an example of metric space $X$ such that $X$ is not complete and every contraction on $X$ has a fixed point.

Here is an elementary proof of Kannan's theorem. 
Proof. Choose $x_{0} \in X$ arbitrarily and define a sequence $\left\{x_{n+1}=T x_{n}, n=\right.$ $0,1,2, \ldots\}$. By (4),

$$
d\left(x_{n+1}, x_{n}\right) \leqslant \frac{K}{1-K} \cdot d\left(x_{n}, x_{n-1}\right) \leqslant \cdots \leqslant\left(\frac{K}{1-K}\right)^{n} \cdot d\left(T x_{0}, x_{0}\right) \rightarrow 0
$$

as $n \rightarrow \infty$. By triangle rule, for $m>n$,

$$
\begin{aligned}
d\left(x_{n}, x_{m}\right) & \leqslant d\left(x_{n}, x_{n+1}\right)+d\left(x_{n+1}, x_{m+1}\right)+d\left(x_{m+1}, x_{m}\right) \\
& \leqslant(K+1) \cdot\left\{d\left(x_{n}, x_{n+1}\right)+d\left(x_{m+1}, x_{m}\right)\right\} \rightarrow 0 \text { as } m>n \rightarrow \infty .
\end{aligned}
$$

Hence $\left\{x_{n}\right\}$ is a Cauchy sequence in a complete metric space $X$ and there exists $p \in X$ such that $x_{n} \rightarrow p \in X$. Since,

$$
\begin{aligned}
d(p, T p) & \leqslant d\left(p, T^{n+1} x\right)+d\left(T^{n+1} x, T p\right) \\
& \leqslant d\left(p, T^{n+1} x\right)+K \cdot\left\{d\left(T^{n} x, T^{n+1} x\right)+d(p, T p)\right\},
\end{aligned}
$$

So

$$
d(p, T p) \leqslant \frac{1}{1-K} \cdot d\left(p, T^{n+1} x\right)+\frac{K}{1-K} \cdot d\left(T^{n} x, T^{n+1} x\right) \rightarrow 0 \text { as } n \rightarrow \infty .
$$

Hence, $p=T p$. Suppose $q$ is another fixed point of $T$. Then

$$
0<d(p, q)=d(T p, T q) \leqslant K \cdot\{d(p, T p)+d(q, T q\}=0,
$$

a contradiction. Hence $T$ has unique fixed point $p \in X$. From (4), we have $\lim _{n \rightarrow \infty} d\left(T^{n} x, p\right)=0$ for any $x \in X$.

Remark 1.9. Theorem 1.8 remains true when (4) is replaced by

$$
d(T x, T y) \leqslant K \cdot\{d(x, T y)+d(y, T x)\} \text { for all } x, y \in X .
$$

The proof is analogous. For more information on mappings satisfying (5), see $[5,10,39]$ and references therein.

Obviously conditions (2) and (4) are independent. Condition (4) is neither stronger nor weaker than the contraction mappings. In particular, the mapping satisfying (4) need not be continuous. In the following examples, the spaces are with the usual metrics.

Example 1.10. Mapping $T x=0$ for $x \leqslant 2$ and $T x=-\frac{1}{2}$ for $x>2$, satisfies (4) with $K=\frac{1}{5}$, and $T$ is not continuous.

Example 1.11. Contraction $T x=\frac{x}{3}, x \in[0,1]$, not satisfied (4) with $K<\frac{1}{2}$, take $x=0$ and $y=1$. If $T$ is a contraction with $L<\frac{1}{3}$, then $T$ satisfies (4) with $K<\frac{1}{2}$.

Example 1.12. The condition (2) with $L=1$, does not imply the existence of a fixed point. The mapping $T x=x+1$ for $x \in \mathbb{R}$ is fixed point free. The condition (4) with $K=\frac{1}{2}$, does not imply the existence of a fixed point. Take the unit circle $S$ on the Euclidean plane and $T z=-z, z \in S$.

There are many generalizations of Theorem 1.6 and Theorem 1.8, and unification of conditions (2) and (4), see [5, 14,31,39], and references therein. The literature of this subject is extensive.

Conclusion. In this part, we have presented elementary proofs of Banach's theorem and Kannan's theorem on a fixed point. 


\section{Asymptotic regularity, continuity and fixed points}

We know many conditions that guarantee the existence of a fixed point, see $[2,17,26]$, and references therein. In this part, we present a very simple situation when the mapping $T$ not only satisfies some conditions of Kannan's type, but it is also continuous and asymptotically regular (as in the Banach theorem).

We recall, asymptotic regularity is a fundamentally important concept in metric fixed point theory, see [2, Chapter IX], and [17, Chapter 9]. It was formally introduced by Browder and Petryshym [7].

Definition 2.1. A mapping $T$ of a metric space $(X, d)$ into itself is said to be asymptotically regular if

$$
\lim _{n \rightarrow \infty} d\left(T^{n} x, T^{n+1} x\right)=0 \text { for all } x \in X .
$$

Obviously, if a mapping $T: X \rightarrow X$ is a contraction or satisfy (4) or (5) with $K<\frac{1}{2}$, then $T$ is asymptotically regular. Asymptotic regularity is also satisfied by other mappings. But already the asymptotic regularity and nonexpansiveness (i.e. $d(T x, T y) \leqslant d(x, y)$ for all $x, y)$, more generally, continuity, are independent.

Example 2.2. Let $B=\left\{x \in \mathbb{R}^{2}:\|x\| \leqslant 1\right\}$ be the closed unit disc in the Euclidean plane and let $T$ be an anticlockwise rotation of $\frac{\pi}{4}$ about the origin of coordinates. Then $T$ is nonexpansive with the origin as the only fixed point and $T$ is not asymptotically regular. Moreover, the sequence defined by $\left\{x_{n+1}=T x_{n}, x_{0}=(1,0)\right\}$ does not converge to zero.

Example 2.3. The mapping $T x=1-x, 0 \leqslant x \leqslant 1$, is continuous, is not a contraction and does not satisfy the condition (4), take $x=0$ and $y=1$. T has a unique fixed point $\frac{1}{2}$, and $d\left(T^{n}(0), T^{n+1}(0)\right) \nrightarrow 0$.

By an averaged mapping we mean one of the form $T_{\lambda}=(1-\lambda) I+\lambda T$, where $0<\lambda<1$ and $I$ is the identity operator. When $T$ is nonexpansive, so is $T_{\lambda}$ and both have the same fixed point set, but $T_{\lambda}$ has more much felicitous asymptotic behavior than the original mapping.

Ishikawa [30] proved the following theorem with no restrictions on the geometry of the Banach space!

Theorem 2.4. If $C$ is a nonempty bounded closed convex subset of a Banach space $X$ and $T: C \rightarrow C$ is nonexpansive, then the mapping $T_{\lambda}$ is asymptotically regular for each $\lambda \in(0,1)$.

It is known [17] that a nonexpansive mapping $T: C \rightarrow C$, acting on weakly compact convex subsets of uniformly convex Banach spaces, has a fixed point. Lin [34] gave an example an asymptotically regulate Lipschitzian mapping acting on a weakly compact convex subset of the Hilbert space $l^{2}$ which has no fixed point.

Asymptotically regular mappings were studied in many papers, in different contexts, for instance [3,8,13,16,19-23,41,48].

In 1974, De Blasi [12] proved the following theorem, see [14]. 
Theorem 2.5. Let $C$ be a nonempty weakly closed subset of a Hilbert space. Suppose that $T: C \rightarrow C$ is continuous, asymptotically regular and satisfies

$$
\|T x-T y\| \leqslant\|x-T x\|+\|y-T y\| \text { for all } x, y \in C .
$$

Then $T$ has a unique fixed point $p \in C$ and $T^{n} x \rightarrow p$ for each $x \in C$.

Now, we prove the following new theorem, which is an extension of previous results.

Theorem 2.6. If $(X, d)$ is a complete metric space and $T: X \rightarrow X$ is a continuous asymptotically regular mapping and if there exists $0 \leqslant M<1$ and $0 \leqslant K<+\infty$ satisfying

$$
d(T x, T y) \leqslant M \cdot d(x, y)+K \cdot\{d(x, T x)+d(y, T y)\} \text { for all } x, y \in X,
$$

then $T$ has a unique fixed point $p \in X$ and $T^{n} x \rightarrow p$ for each $x \in X$.

Proof. Choose $x_{0} \in X$ arbitrarily and define a sequence $\left\{x_{n+1}=T x_{n}, n=\right.$ $0,1,2, \ldots\}$. According to asymptotic regularity, by triangle rule and (6), we get for any $n$ and any $k>0$,

$$
\begin{aligned}
d\left(x_{n+k}, x_{n}\right) \leqslant & d\left(x_{n+k}, x_{n+k+1}\right)+d\left(x_{n+k+1}, x_{n+1}\right)+d\left(x_{n+1}, x_{n}\right) \\
\leqslant & d\left(x_{n+k}, x_{n+k+1}\right)+M \cdot d\left(x_{n+k}, x_{n}\right) \\
& +K \cdot\left\{d\left(x_{n+k}, x_{n+k+1}\right)+d\left(x_{n}, x_{n+1}\right)\right\}+d\left(x_{n+1}, x_{n}\right),
\end{aligned}
$$

so

$$
(1-M) \cdot d\left(x_{n+k}, x_{n}\right) \leqslant(K+1) \cdot\left\{d\left(x_{n+k}, x_{n+k+1}\right)+d\left(x_{n}, x_{n+1}\right)\right\} \rightarrow 0,
$$

as $n \rightarrow \infty$. This shows that $\left\{x_{n}\right\}$ is a Cauchy sequence in complete space $X$. There exists $p \in X$ such that $x_{n} \rightarrow p$. Because $T$ is continuous and $x_{n+1}=T x_{n}$, it follows that $p=T p$. Suppose $q$ is another fixed point of $T$. Then

$$
\begin{aligned}
0<d(p, q) & =d(T p, T q) \leqslant M \cdot d(p, q)+(K+1) \cdot\{d(p, T p)+d(q, T q)\} \\
& =M \cdot d(p, q)<d(p, q),
\end{aligned}
$$

a contradiction. Hence $T$ has unique fixed point $p \in X$. Because

$$
\begin{aligned}
d\left(T^{n} x, p\right)=d\left(T^{n} x, T^{n} p\right) \leqslant & d\left(T^{n} x, T^{n+1} x\right)+d\left(T^{n+1} x, T^{n+1} p\right) \\
\leqslant & d\left(T^{n} x, T^{n+1} x\right)+M \cdot d\left(T^{n} x, T^{n} p\right) \\
& +K \cdot d\left(T^{n} x, T^{n+1} x\right),
\end{aligned}
$$

So

$$
(1-M) \cdot d\left(T^{n} x, p\right) \leqslant(K+1) \cdot d\left(T^{n} x, T^{n+1} x\right) \rightarrow 0 \text { as } n \rightarrow \infty .
$$

This shows that $T^{n} x \rightarrow p$ for any $x \in X$.

Remark 2.7. If $M \geqslant 0, K \geqslant 0$ and $M+2 K<1$, then assumptions of continuity and asymptotic regularity are not necessary for the thesis to hold. If $0 \leqslant M<1$ and $0 \leqslant K<1$, then the continuity assumption is not necessary for the thesis to hold, see $[25,39]$. 
Remark 2.8. Theorem 2.4 remains true when condition (6) is replaced by

$$
d(T x, T y) \leqslant M \cdot d(x, y)+K \cdot\{d(y, T x)+d(x, T y)\} \text { for all } x, y \in X,
$$

see $[5,10,49]$.

Example 2.9. Let $X=[0,1] \cup\left[\frac{3}{2}, \frac{5}{3}\right]$ with the usual metric $d(x, y)=|x-y|$ and $T: X \rightarrow X$ be given by $T x=0$, if $0 \leqslant x \leqslant 1$ and $T x=1$, if $\frac{3}{2} \leqslant x \leqslant \frac{5}{3}$. Then, $T 0=0$ and

(a) $T$ does not satisfy the Banach theorem, take $x=1$ and $y=\frac{3}{2}$;

(b) $T$ does not satisfy the Kannan theorem, take $x=0$ and $y=\frac{3}{2}$;

(c) $T$ is asymptotically regular;

(d) $T$ is continuous;

(e) $T$ satisfies (6) with $K=2$ and any $0 \leqslant M<1$.

Indeed, if $x, y \in[0,1]$ or $x, y \in\left[\frac{3}{2}, \frac{5}{3}\right]$, then $d(T x, T y)=0$, when the condition (6) is obviously satisfied. If $x \in[0,1]$ and $y \in\left[\frac{3}{2}, \frac{5}{3}\right]$, then $d(T x, T y)=1$ and $d(x, y) \geqslant \frac{1}{2}, d(x, T x)+d(y, T y) \geqslant x+y-1 \geqslant \frac{1}{2}$.

Therefore,

$d(T x, T y) \leqslant M \cdot d(x, y)+2 \cdot\{d(x, T x)+d(y, T y)\}$ for all $x, y \in X$,

and any $0 \leqslant M<1$.

When $M=0$, then from Theorem 2.6 we have a significant extension of Banach's theorem in a new direction:

Corollary 2.10. Let $(X, d)$ be a complete metric space and $T: X \rightarrow X$ be a continuous and asymptotically regular mapping satisfying (4) with $0 \leqslant K<$ $+\infty$ (especially, $K \geqslant 1$ ), then $T$ has a unique fixed point $p \in X$ and $T^{n} x \rightarrow p$ for each $x \in X$.

Remark 2.11. Note that each contraction with constant $L<1$ satisfies (4) with constant $K=\frac{L}{1-L}$. Indeed, for all $x, y \in X$,

$$
d(T x, T y) \leqslant L \cdot d(x, y) \leqslant L \cdot\{d(x, T x)+d(T x, T y)+d(T y, y)\},
$$

so

$$
d(T x, T y) \leqslant \frac{L}{1-L} \cdot\{d(x, T x)+d(y, T y)\}
$$

Therefore, all contractions satisfy the assumptions of Corollary 2.10.

Example 2.12. Let $T:\left[0, \frac{\pi}{2}\right] \rightarrow \mathbb{R}$ be defined by $T x=\cos x$. $T$ is not a contraction. Suppose there exists $L \in(0,1)$ such that

$$
\left|\frac{\cos x-\cos y}{x-y}\right| \leqslant L \text { for all } x \neq y \text {. }
$$

Letting $y \rightarrow x$, we get $|\sin x| \leqslant L$ for all $x, y \in\left[0, \frac{\pi}{2}\right]$, which is false. For $x, y \in\left[0, \frac{\pi}{2}\right]$ we have

$$
\begin{aligned}
|\cos x-\cos y| & =\left|-2 \sin \frac{x+y}{2} \sin \frac{x-y}{2}\right| \leqslant|x-y| \\
& \leqslant|x-1|+|y-1| \leqslant|x-\cos x|+|y-\cos y| .
\end{aligned}
$$


Obviously $T$ is continuous and if $x_{n}=T^{n} x=\cos x_{n-1}, x_{0} \in\left[0, \frac{\pi}{2}\right]$, then

$$
\left|T^{n+1} x-T^{n} x\right|=\left|\cos x_{n}-\cos x_{n-1}\right|=\left|\sin c_{n}\right| \cdot\left|x_{n}-x_{n-1}\right|,
$$

where a point $c_{n}$ is between $x_{n}$ and $x_{n-1}$, so $\left|\sin c_{n}\right|<1$ for $n=1,2, \ldots$ Hence $T$ is an asymptotically regular mapping. Corollary 2.10 guarantees that $T$ has a unique fixed point $p \in\left[0, \frac{\pi}{2}\right]$ and $p=\lim _{n \rightarrow \infty} x_{n}$, where $x_{n+1}=\cos x_{n}$, $x_{0} \in\left[0, \frac{\pi}{2}\right]$. Approximate solution of the equation $x=\cos x$ is $p \approx 0.739$.

Example 2.13. Let $X=\mathbb{R}$ be with the usual metric and let $T$ be defined as follows: $T 0=0$ and $T x=\frac{x}{2} \sin \frac{1}{x}$ if $x \neq 0$. Taking $x=\frac{2}{\pi}$ and $y=\frac{2}{3 \pi}$, we obtain

$$
|T x-T y|=\frac{8}{3 \pi}>\frac{4}{3 \pi}=|x-y| .
$$

Taking $x=0$ and $y=\frac{2}{\pi}$, we have

$$
\left|T 0-T\left(\frac{2}{\pi}\right)\right|=\frac{1}{\pi}=|0-T 0|+\left|\frac{2}{\pi}-T\left(\frac{2}{\pi}\right)\right|,
$$

so there is no universal constant $K<1$ satisfying (4), therefore Corollary 2.10 is also some extension of Theorem 3.1 from [25]. On the other hand,

$|T x-T y| \leqslant|T x|+|T y| \leqslant \frac{|x|}{2}+\frac{|y|}{2}=\left|x-\frac{x}{2}\right|+\left|y-\frac{y}{2}\right| \leqslant|x-T x|+|y-T y|$

for all $x, y \in \mathbb{R}$. Obviously $T$ is asymptotically regular and continuous, therefore, all assumptions of the Corollary 2.10 are fulfilled.

Remark 2.14. For clarity of this presentation we omit discussion in $b$-metric spaces (see [33]) and $G$-metric spaces (see [1]) and consideration of semigroups $[20]$.

Conclusion. In this part, we presented a new extension of Banach's theorem with examples.

\section{Fixed point theorems in preordered sets}

An interplay between the order and metrical structure of the space turned out to be very fruitful. In Refs. [36,37], we find an analogue of Banach theorem in partially ordered sets, further extensions are contained in $[24,42,43]$. In all these works, the mapping considered are monotone. For such mappings one of the fundamental results in fixed-point theory is the classical Knaster-Tarski theorem (also known as the Abian-Brown theorem), see [26], [38]. Recently, Espínola and Wiśnicki [15] studied the problem whether the classical Kirk's theorem for nonexpansive mappings (see [17]) still holds for monotone-nonexpansive mappings. They proved in some partially ordered sets a general theorem which guarantees the existence of a fixed point for monotone mappings (which need not be either monotone-nonexpansive nor continuous), and which does not impose any conditions on the Banach space.

An interesting reference with many applications of the fixed point theory of monotone mappings is [9]. 
In this section, we extend Corollary 2.10 on preordered metric spaces, where a preordered binary relation is weaker than a partial order. The key feature in this theorem is that the Kannan's type condition on the map is only assumed to hold on elements that are comparable but not on the entire set on which they are defined, see Example 3.9.

Definition 3.1. Let $X \neq \emptyset$ be a set. Binary relation $\preccurlyeq$ on $X$ is

(a) reflexive if $x \preccurlyeq x$ for all $x \in X$,

(b) transitive if $x \preccurlyeq z$ for all $x, y, z \in X$ such that $x \preccurlyeq y$ and $y \preccurlyeq z$.

A reflexive and transitive relation on $X$ is a preordered on $X$. In such case ( $X, \preccurlyeq)$ is a preordered space. Write $x \prec y$ when $x \preccurlyeq y$ and $x \neq y$. We will say that $x, y \in X$ are comparable whenever $x \preccurlyeq y$ or $y \preccurlyeq x$.

Example 3.2. Let $\preccurlyeq$ be the binary relation on $\mathbb{R}$ given by

$$
x \preccurlyeq y \Leftrightarrow(x=y \text { or } x<y \leqslant 0) .
$$

Then $\preccurlyeq$ is a partial order (and so preordered) on $\mathbb{R}$, but it is different from $\leqslant$.

Definition 3.3. A preordered metric space is a triple $(X, d, \preccurlyeq)$ where $(X, d)$ is a metric space and $\preccurlyeq$ is a preordered on $X$.

One of the most important hypothesis that we shall use in this section is the monotonicity of the involved mappings.

Definition 3.4. Let $\preccurlyeq$ be a binary relation on $X$. A map $T: X \rightarrow X$ is monotone if $T x \preccurlyeq T y$ whenever $x \preccurlyeq y$.

The following result is the extension of Corollary 2.10 to Kannan's type mappings on preordered metric spaces.

Theorem 3.5. Let $(X, d, \preccurlyeq)$ be a preordered metric space and let $T: X \rightarrow X$ be a mapping. Suppose that the following conditions hold:

(i) $(X, d)$ is complete,

(ii) $T$ is monotone,

(iii) $T$ is continuous,

(iv) there exists $x_{0} \in X$ such that $x_{0} \preccurlyeq T x_{0}$,

(v) $T$ is asymptotically regular, i.e. $\lim _{n \rightarrow \infty} d\left(T^{n} x, T^{n+1} x\right)=0$ for all $x \in X$,

(vi) for all $x, y \in X$ with $x \preccurlyeq y$,

$$
d(T x, T y) \leqslant K \cdot\{d(x, T x)+d(y, T y)\} \text { for some } 0 \leqslant K<+\infty .
$$

Then there exists a fixed point of $T$, and it is unique, say $u$, if

$$
\forall(x, y) \in X \times X \exists_{w \in X} \quad(x \preccurlyeq w \text { and } y \preccurlyeq w) .
$$

Moreover, for each $x_{0} \in X$ such that $x_{0} \preccurlyeq T x_{0}$, the sequence $\left\{T^{n} x_{0}\right\}$ of iterates converges to $u$.

Proof. Let $x_{0} \in X$ be a point satisfying (iv), that is, $x_{0} \preccurlyeq T x_{0}$. We define a sequence $\left\{x_{n}\right\} \subset X$ as follows

$$
x_{n}=T x_{n-1}, n \geqslant 1 .
$$


Regarding that $T$ is a monotone mapping together with (10) we have

$$
x_{0} \preccurlyeq T x_{0}=x_{1} \text { implies } x_{1}=T x_{0} \preccurlyeq T x_{1}=x_{2} \text {. }
$$

Inductively, we obtain

$$
x_{0} \preccurlyeq x_{1} \preccurlyeq x_{2} \cdots \preccurlyeq x_{n-1} \preccurlyeq x_{n} \preccurlyeq x_{n+1} \preccurlyeq \cdots
$$

Now, by triangle rule and asymptotic regularity, for $m>n$, we get

$$
\begin{aligned}
& d\left(x_{n}, x_{m}\right) \leqslant d\left(x_{n}, x_{n+1}\right)+d\left(x_{n+1}, x_{m+1}\right)+d\left(x_{m+1}, x_{m}\right) \\
& \quad \leqslant d\left(x_{n}, x_{n+1}\right)+K \cdot\left\{d\left(x_{n}, x_{n+1}\right)+d\left(x_{m}, x_{m+1}\right)\right\}+d\left(x_{m}, x_{m+1}\right) \\
& \quad=(K+1) \cdot\left\{d\left(x_{n}, x_{n+1}\right)+d\left(x_{m}, x_{m+1}\right)\right\} \rightarrow 0
\end{aligned}
$$

as $m>n \rightarrow \infty$. This implies that $\left\{x_{n}\right\}$ is a Cauchy sequence in $X$. From the completeness of $X$ there exists $u \in X$ such that $x_{n} \rightarrow u$. Because $T$ is continuous and $x_{n+1}=T x_{n}$, it follows that $u=T u$.

To prove uniqueness, we assume that $v \in X$ is another fixed point of $T$ such that $u \neq v$. By hypothesis, there exists $w \in X$ such that $u \preccurlyeq w$ and $v \preccurlyeq w$.

Let $\left\{w_{n}=T w_{n-1}\right\}$ be the sequence of successive approximations of $T$ based on $w_{0}=w$. As $T$ is monotone, $v=T v \preccurlyeq T w=w_{1}$ and $u=T u \preccurlyeq$ $T w=w_{1}$. By induction, $v \preccurlyeq w_{n}$ and $u \preccurlyeq w_{n}$ for all $n \geqslant 0$.

Case 1. If $v=w_{n_{0}}$ for some $n_{0} \geqslant 0$, then $v=T v=T w_{n_{0}}=w_{n_{0}+1}$ and by induction, $w_{n}=v$ for all $n \geqslant n_{0}$, so $w_{n} \rightarrow v$.

Case 2. If $v \prec w_{n}$ for all $n \geqslant 0$, then

$$
\begin{aligned}
d\left(v, w_{n+1}\right) & =d\left(T v, T w_{n}\right) \leqslant K \cdot\left\{d(v, T v)+d\left(w_{n}, T w_{n}\right)\right\} \\
& =K \cdot\left\{d(v, T v)+d\left(T^{n} w, T^{n+1} w\right)\right\} \rightarrow 0,
\end{aligned}
$$

as $n \rightarrow \infty$, by asymptotic regularity. Hence $w_{n} \rightarrow v$.

Thus $w_{n} \rightarrow v$ and $w_{n} \rightarrow u$. The uniqueness of the limit concludes that $u=v$, so $T$ has a unique fixed point.

Remark 3.6. Theorem 3.5 remains true when condition (6) is satisfied (with $0 \leqslant M<1$ and $0 \leqslant K<+\infty)$ in place of condition (8). Then in Case 2 we have an estimate:

$$
\begin{aligned}
d\left(v, w_{n+1}\right) & =d\left(T v, T w_{n}\right) \\
& \leqslant M \cdot d\left(v, w_{n}\right)+K \cdot\left\{d(v, T v)+d\left(w_{n}, T w_{n}\right)\right\} \\
& \leqslant M \cdot\left\{d\left(v, w_{n+1}\right)+d\left(w_{n+1}, w_{n}\right)\right\}+K \cdot\left\{d(v, T v)+d\left(w_{n}, T w_{n}\right)\right\}
\end{aligned}
$$

SO

$(1-M) \cdot d\left(v, w_{n+1}\right) \leqslant M \cdot d\left(w_{n+1}, w_{n}\right)+K \cdot\left\{d(v, T v)+d\left(w_{n}, T w_{n}\right)\right\} \rightarrow 0$

as $n \rightarrow \infty$. Hence $w_{n} \rightarrow v$. Then, we act as in the proof of Theorem 3.5.

After the appearance of the Ran and Reurings' result [37], Nieto and Rodríguez-López [36] changed the continuity of the mapping $T$ with the condition nondecreasing regularity (Definition 3.7). Now, we exchanged the continuity of the mapping $T$ with the condition nondecreasing regularity and we obtain in preordered metric spaces an analogue of [25, Theorem 3.1]. 
Definition 3.7. Let $(X, d)$ be a metric space, let $A \subset X$ be a nonempty subset and let $\preccurlyeq$ be a binary relation on $X$. Then triple $(A, d, \preccurlyeq)$ is said to be nondecreasing regular if for all sequence $\left\{x_{n}\right\} \subset A$ such that $\left\{x_{n}\right\} \rightarrow x \in A$ and $x_{n} \preccurlyeq x_{n+1}$ for all $n \in \mathbb{N}$, we have that $x_{n} \preccurlyeq x$ for all $n \in \mathbb{N}$.

Theorem 3.8. Let $(X, d, \preccurlyeq)$ be a preordered metric space and let $T: X \rightarrow X$ be a mapping. Suppose that the following conditions hold:

(i) $(X, d)$ is complete,

(ii) $T$ is monotone,

(iii) $(X, d, \preccurlyeq)$ is nondecreasing regular,

(iv) there exists $x_{0} \in X$ such that $x_{0} \preccurlyeq T x_{0}$,

(v) $T$ is asymptotically regular, i.e. $\lim _{n \rightarrow \infty} d\left(T^{n} x, T^{n+1} x\right)=0$ for all $x \in X$,

(vi) for all $x, y \in X$ with $x \preccurlyeq y$,

$$
d(T x, T y) \leqslant K \cdot\{d(x, T x)+d(y, T y)\} \text { for some } 0 \leqslant K<1 .
$$

Then there exists a fixed point of $T$, and it is unique, say u, if (9) is satisfied. Moreover, for each $x_{0} \in X$ such that $x_{0} \preccurlyeq T x_{0}$, the sequence $\left\{T^{n} x_{0}\right\}$ of iterates converges to $u$.

Proof. Following the proof of Theorem 3.5, we have a monotone (nondecreasing) sequence $\left\{x_{n}=T x_{n-1}\right\}$ which is convergent to $u \in X$. Due to (iii), we have $x_{n} \preccurlyeq u$ for all $n \geqslant 1$. Now, we show that $u$ is a fixed point of $T$. Fix an $\varepsilon>0$. Since $T^{n} x_{0} \rightarrow u$, given $\frac{\varepsilon}{2}>0$, there exists $n_{1} \in \mathbb{N}$ such that for all $n \geqslant n_{1}$,

$$
d\left(T^{n} x_{0}, u\right)<\frac{\varepsilon}{2}(1-K) \text { and } d\left(T^{n} x_{0}, T^{n+1} x_{0}\right)<\frac{\varepsilon}{2}(1-K) .
$$

Taking $n \geqslant n_{1}$ and using that $T^{n} x_{0} \preccurlyeq u$ for all $n \in \mathbb{N}$, we get

$$
\begin{aligned}
d(T u, u) & \leqslant d\left(T u, T^{n+1} x_{0}\right)+d\left(T^{n+1} x_{0}, u\right) \\
& \leqslant K \cdot\left\{d(u, T u)+d\left(T^{n} x_{0}, T^{n+1} x_{0}\right)\right\}+d\left(T^{n+1} x_{0}, u\right),
\end{aligned}
$$

so

$$
\begin{aligned}
d(T u, u) & \leqslant \frac{K}{1-K} d\left(T^{n} x_{0}, T^{n+1} x_{0}\right)+\frac{1}{1-K} d\left(T^{n+1} x_{0}, u\right) \\
& <\frac{1}{1-K} d\left(T^{n} x_{0}, T^{n+1} x_{0}\right)+\frac{1}{1-K} d\left(T^{n+1} x_{0}, u\right)<\varepsilon .
\end{aligned}
$$

In consequence, since $\varepsilon>0$ is arbitrary, $d(T u, u)=0$. Hence $u=T u$. Uniqueness of $u$ can be observed as in the proof of Theorem 3.5.

Observe that condition (4) with $0 \leqslant K<1$, see [25, Theorem 3.1], is slightly stronger than condition (vi) of Theorem 3.8, which only requires the inequality for comparable points, that is, for all $x, y \in X$ such that $x \preccurlyeq y$ or $y \preccurlyeq x$.

Example 3.9. Let $X=[-1,1]$ be endowed with the metric $d(x, y)=|x-y|$ for all $x, y \in X$. Consider on $X$ the partial order

$$
x \preccurlyeq y \Longleftrightarrow(x=y \text { or } x<y \leqslant 0) .
$$


Define $T: X \rightarrow X$ by $T x=-\frac{x}{4}$ for $-1 \leqslant x \leqslant 0$ and $T x=\frac{9}{10} x$ for $0<x \leqslant 1$. Obviously mapping $T$ is asymptotically regular. Let $x, y \in X$ be such that $x \preccurlyeq y$. If $x=y$, then

$$
d(T x, T y)=0 \leqslant \frac{1}{3} \cdot\{d(x, T x)+d(y, T y)\}
$$

trivially holds. Assume that $x \neq y$. Then $x<y \leqslant 0$. Hence

$$
d(T x, T y) \leqslant \frac{1}{4}(|x|+|y|) \quad \text { and } \quad d(x, T x)+d(y, T y) \geqslant \frac{3}{4}(|x|+|y|) .
$$

Thus

$$
d(T x, T y) \leqslant \frac{1}{3} \cdot\{d(x, T x)+d(y, T y)\}
$$

for all $x, y \in[-1,0]$. Hence (vi) holds. However, condition (4) with $0 \leqslant K<1$ is false in this case because if $x=0$ and $y=1$, then $d(T(0), T(1))=\frac{9}{10}>$ $\frac{1}{10}=d(0, T(0))+d(1, T(1))$.

In the next example, we have shown that if condition (9) in Theorems 3.5 and 3.8 fails, it is possible to find examples of functions $T$ with more than one fixed point.

Example 3.10. Let $X=\{(1,0),(0,1)\} \subset \mathbb{R}^{2}$, and consider the order

$$
(x, y) \preccurlyeq(z, t) \Longleftrightarrow(x \leqslant z \text { and } y \leqslant t) .
$$

Thus, $(X, \preccurlyeq)$ is a partially ordered set, whose different elements are not comparable. The metric space $(X, d)$ with the Euclidean distance is a complete metric space. The identity map $T(x, y)=(x, y)$ is trivially continuous, asymptotically regular, non-decreasing and condition

$$
d(T(x, y), T(z, t)) \leqslant K \cdot\{d((x, y), T(x, y))+d((z, t), T(z, t))\},
$$

holds for any $0 \leqslant K<+\infty$, since elements in $X$ are only comparable to themselves. Moreover, $(1,0) \preccurlyeq T(1,0)=(1,0)$. In this case, there are two fixed points in $X$. Hypotheses in Theorem 3.5 hold. Theorem 3.8 is also applicable since if $\left\{\left(x_{n}, y_{n}\right)\right\} \subset X$ is a monotone (nondecreasing) sequence converging to $(x, y) \in X$, then necessarily $\left.\left(x_{n}, y_{n}\right)\right\}$ is a constant sequence and $\left(x_{n}, y_{n}\right)=(x, y)$ for all $n \in \mathbb{N}$, so the limit $(x, y)$ is an upper bound for all the terms in the sequence.

This shows that conditions in Theorems 3.5 and 3.8 do not imply uniqueness of the fixed point.

In this example, condition (9) does not hold, since given two different elements in $X$, there is no upper bound of them. In this case, $T$ may have more than one fixed point.

Conclusion. In this section, we discussed the extension of Banach's theorem in preordered metric spaces. 


\section{Random fixed-point theorems}

An interesting aspect of the nonlinear analysis is to randomize deterministic fixed-point theorems of nonlinear mappings. The study of fixed-point theorems for random operators was initiated by the Prague school of probability research. The first results were studied in 1955-1956 by Špaček and Hanš in the context of Fredholm integral equations with random kernel, see for instance [45]. In a separable metric space, random fixed-point theorems for contraction mappings were proved by Hanš [27] (for some set-valued mappings see [40]).

In many cases, the mathematical models or equations used to describe phenomena in biology, physics, engineering contain certain parameters whose values are unknown. Then, it is more realistic to consider such equations as random operator equations. These equations are much more difficult to handle mathematically than deterministic equations [6].

It has been shown that when the underlying measurable space $(\Omega, \Sigma)$ is a Suslin family (see [47] for definitions), a deterministic fixed-point theorem may, in general, correspond to a random fixed-point theorem. However, it is unknown if the same is true when the measurable space $(\Omega, \Sigma)$ is not a Suslin family.

Nieto et al. [35] proved the random version in partially ordered metric spaces of the classical Banach contraction principle. In this section, we will prove some random fixed point theorems for single-valued operators which are asymptotically regular and satisfies some Kannan's type conditions.

Let $(\Omega, \Sigma)$ be a measurable space with $\Sigma$ a $\sigma$-algebra of subsets of $\Omega$. For a metric space $(X, d)$, we denote by $C L(X)$ the family of all nonempty closed subsets of $X$.

Definition 4.1. A set-valued operator $F: \Omega \rightarrow 2^{X}$ is called $\Sigma$-measurable if for any open subset $B$ of $X$, the set $F^{-1}(B)=\{\omega \in \Omega: F(\omega) \cap B \neq \emptyset\}$ belongs to $\Sigma$.

Definition 4.2. A measurable (single-valued) operator $x: \Omega \rightarrow X$ is called a selector for a measurable set-valued operator $F: \Omega \rightarrow 2^{X}$ if $x(\omega) \in F(\omega)$ for all $\omega \in \Omega$.

Definition 4.3. A mapping $T: \Omega \times X \rightarrow X$ is called a random operator if for each $x \in X$, the map $T(\cdot, x): \Omega \rightarrow X$ is measurable.

Definition 4.4. A measurable operator $x: \Omega \rightarrow X$ is said to be a random fixed point of random operator $T: \Omega \times X \rightarrow X$ if $T(\omega, x(\omega))=x(\omega)$ for all $\omega \in \Omega$.

Equivalently, it is a measurable selection for the set-valued map Fix T : $\Omega \rightarrow 2^{X}$ defined by Fix $T(\omega)=\{x \in X: T(\omega, x)=x\}$.

We recall, a random mapping $T: \Omega \times X \rightarrow X$ is said to be continuous if for each fixed $\omega \in \Omega$, the map $T(\omega, \cdot): X \rightarrow X$ has this particular property.

We will list the following results related to the concept of measurability.

Theorem $4.5[29]$. Let $(\Omega, \Sigma)$ be a measurable space, $X$ be a separable metric space and $Y$ a metric space. If $T: \Omega \times X \rightarrow Y$ is measurable in $\omega \in \Omega$ and 
continuous in $x \in X$, respectively, and if $x: \Omega \rightarrow X$ is measurable, then $T(\cdot, x(\cdot)): \Omega \rightarrow X$ is a measurable.

Theorem 4.6 [47]. Let $(\Omega, \Sigma)$ be a measurable space, $(Y, d)$ be a Polish space (i.e. complete and separable metric space) and $F: \Omega \rightarrow C L(Y)$ a measurable map. Then $F$ has a measurable selection.

Definition 4.7. Let $(\Omega, \Sigma)$ be a measurable space and $(X, d)$ be a metric space. A random operator $T: \Omega \times X \rightarrow X$ is said to be asymptotically regular if for each fixed $\omega \in \Omega$,

$$
\lim _{n \rightarrow \infty} d\left(T^{n}(\omega, x), T^{n+1}(\omega, x)\right)=0
$$

for all $x \in X$. Here $T^{n}(\omega, x)$ is the value at $x$ of the $n$th iterate of the map $T(\omega, \cdot)$, i.e. $T^{n}(\omega, x)=T\left(\omega, T^{n-1}(\omega, x)\right)$.

The following result is the randomization of Theorem 2.6.

Theorem 4.8. Let $(\Omega, \Sigma)$ be a measurable space and $(X, d)$ be a Polish space. If $T: \Omega \times X \rightarrow X$ is a random continuous operator which is asymptotically regular, and exists functions $M: \Omega \rightarrow[0,1)$ and $K: \Omega \rightarrow[0, \infty)$ such that for each $\omega \in \Omega$,

$$
\begin{aligned}
& d(T(\omega, x), T(\omega, y)) \\
& \quad \leqslant M(\omega) \cdot d(x, y)+K(\omega) \cdot\{d(x, T(\omega, x))+d(y, T(\omega, y))\}
\end{aligned}
$$

for all $x, y \in X$, then $T$ has a unique random fixed point.

(We do not assume the measurability of the functions $M(\cdot)$ and $K(\cdot)$.)

Proof. Fix a measurable function $x_{0}: \Omega \rightarrow X$. If for each $\omega \in \Omega, T\left(\omega, x_{0}(\omega)\right)$ $=x_{0}(\omega)$, then $x_{0}$ is a random fixed point of $T$. Suppose that, for some $\omega \in \Omega$, $T\left(\omega, x_{0}(\omega)\right) \neq x_{0}(\omega)$. We define the sequence

$$
y_{0}(\omega)=x_{0}(\omega) \text { and } y_{n}(\omega)=T\left(\omega, y_{n-1}(\omega)\right)=T^{n}\left(\omega, x_{0}(\omega)\right) \text {, }
$$

for all $\omega \in \Omega$ and integers $n \geqslant 1$. Using (11), by triangle rule and asymptotic regularity, we get for $m>n$,

$$
\begin{aligned}
d\left(y_{n}(\omega), y_{m}(\omega)\right) & \\
\leqslant & d\left(y_{n}(\omega), y_{n+1}(\omega)\right)+d\left(y_{n+1}(\omega), y_{m+1}(\omega)\right)+d\left(y_{m+1}(\omega), y_{m}(\omega)\right) \\
\leqslant & d\left(y_{n}(\omega), y_{n+1}(\omega)\right)+M(\omega) \cdot d\left(y_{n}(\omega), y_{m}(\omega)\right) \\
\quad & +K(\omega) \cdot\left\{d\left(y_{n}(\omega), y_{n+1}(\omega)\right)+d\left(y_{m}(\omega), y_{m+1}(\omega)\right)\right\}+d\left(y_{m+1}(\omega), y_{m}(\omega)\right),
\end{aligned}
$$

So

$$
\begin{aligned}
& (1-M(\omega)) \cdot d\left(y_{n}(\omega), y_{m}(\omega)\right) \\
& \quad \leqslant(K(\omega)+1) \cdot\left\{d\left(y_{n}(\omega), y_{n+1}(\omega)\right)+d\left(y_{m}(\omega), y_{m+1}(\omega)\right)\right\} \rightarrow 0,
\end{aligned}
$$

as $m>n \rightarrow \infty$. Hence $\left\{y_{n}(\omega)\right\}_{n}$ is a Cauchy sequence for every $\omega \in \Omega$. Since $X$ is a complete space there exists $y_{*}(\omega) \in X, \omega \in \Omega$, such that $y_{*}(\omega)=$ $\lim _{n \rightarrow \infty} y_{n}(\omega)$. Since $y_{0}(\cdot)$ is measurable, then $y_{1}(\cdot)$ is measurable. Hence, by induction, we can easily prove that for each $n \in \mathbb{N}$, the function $\omega \rightarrow y_{n}(\omega)$ is measurable. The mapping $y_{*}: \Omega \rightarrow X$ is the pointwise limit of measurable mappings, so it is measurable. 
Now, we show that $y_{*}$ is a random fixed point of $T$, i.e. $y_{*}(\omega)=$ $T\left(\omega, y_{*}(\omega)\right), \omega \in \Omega$. It is clear, for each $\omega \in \Omega$, by asymptotic regularity, we get

$$
\lim _{n \rightarrow \infty} d\left(y_{n}(\omega), y_{n+1}(\omega)\right)=\lim _{n \rightarrow \infty} d\left(T^{n}\left(\omega, x_{0}(\omega)\right), T^{n+1}\left(\omega, x_{0}(\omega)\right)\right)=0,
$$

and because $T(\omega, \cdot)$ is continuous,

$$
d\left(y_{*}(\omega), T\left(\omega, y_{*}(\omega)\right)\right)=\lim _{n \rightarrow \infty} d\left(y_{n}(\omega), T\left(\omega, y_{n}(\omega)\right)\right)=0 .
$$

Thus,

$$
y_{*}(\omega)=T\left(\omega, y_{*}(\omega)\right) \text { for each } \omega \in \Omega .
$$

The uniqueness of the random fixed point follows from the uniqueness of $y_{*}(\omega)$ for every $\omega \in \Omega$. Suppose $z_{*}(\omega)=T\left(\omega, z_{*}(\omega)\right)$ is another random fixed point of $T$, then by (11),

$$
\begin{aligned}
0< & d\left(y_{*}(\omega), z_{*}(\omega)\right)=d\left(T\left(\omega, y_{*}(\omega)\right), T\left(\omega, z_{*}(\omega)\right)\right) \\
\leqslant & M(\omega) \cdot d\left(y_{*}(\omega), z_{*}(\omega)\right) \\
& +K(\omega) \cdot\left\{d\left(y_{*}(\omega), T\left(\omega, y_{*}(\omega)\right)\right)+d\left(z_{*}(\omega), T\left(\omega, z_{*}(\omega)\right)\right)\right\} \\
= & M(\omega) \cdot d\left(y_{*}(\omega), z_{*}(\omega)\right)<d\left(y_{*}(\omega), z_{*}(\omega)\right),
\end{aligned}
$$

a contradiction. Hence $y_{*}(\omega)=z_{*}(\omega)$.

Corollary 4.9. Let $(\Omega, \Sigma)$ be a measurable space and $(X, d)$ be a Polish space. If $T: \Omega \times X \rightarrow X$ is a random operator, and exists a function $M: \Omega \rightarrow[0,1)$ such that for each $\omega \in \Omega$,

$$
d(T(\omega, x), T(\omega, y)) \leqslant M(\omega) \cdot d(x, y)
$$

for all $x, y \in X$, then $T$ has a unique random fixed point.

Corollary 4.10. Let $(\Omega, \Sigma)$ be a measurable space and $(X, d)$ be a Polish space. If $T: \Omega \times X \rightarrow X$ is a random continuous operator which is asymptotically regular, and exists a function $K: \Omega \rightarrow[0, \infty)$ such that for each $\omega \in \Omega$,

$$
d(T(\omega, x), T(\omega, y)) \leqslant K(\omega) \cdot\{d(x, T(\omega, x))+d(y, T(\omega, y))\}
$$

for all $x, y \in X$, then $T$ has a unique random fixed point.

Now, we establish a random version of some fixed-point theorem in preordered metric spaces.

Theorem 4.11. Let $(\Omega, \Sigma)$ be a measurable space, $(X, d, \preccurlyeq)$ be a Polish preordered metric space and let $T: \Omega \times X \rightarrow X$ be a mapping. Suppose that the following conditions hold:

(i) $T$ is a continuous random operator,

(ii) for each $\omega \in \Omega$, the function $T(\omega, \cdot)$ is monotone operator, i.e.

$$
(x, y \in X \text { and } x \preccurlyeq y) \Longrightarrow T(\omega, x) \preccurlyeq T(\omega, y) \text {, }
$$

(iii) there exists a random variable $x_{0}: \Omega \rightarrow X$ with

$$
x_{0}(\omega) \preccurlyeq T\left(\omega, x_{0}(\omega)\right) \text { or } x_{0}(\omega) \succcurlyeq T\left(\omega, x_{0}(\omega)\right) \text { for each } \omega \in \Omega \text {, }
$$

(iv) $T$ is asymptotically regular, 
(v) there exists a function $K: \Omega \rightarrow[0, \infty)$ such that for each $\omega \in \Omega$,

$$
d(T(\omega, x), T(\omega, y)) \leqslant K(\omega) \cdot\{d(x, T(\omega, x))+d(y, T(\omega, y))\}
$$

for every comparable $x, y \in X$, i.e. $x \preccurlyeq y$ or $y \preccurlyeq x$.

Then there exists a random variable $x: \Omega \rightarrow X$ which is a random fixed point of $T$, and it is unique if

for every $x, y \in X$, there exists $z \in X$ that is comparable to $x$ and $y$.

Proof. Fix a measurable function $x_{0}: \Omega \rightarrow X$. If for each $\omega \in \Omega, T\left(\omega, x_{0}(\omega)\right)$ $=x_{0}(\omega)$, then $x_{0}$ is a random fixed point of $T$. Suppose that, for some $\omega \in \Omega$, $T\left(\omega, x_{0}(\omega)\right) \neq x_{0}(\omega)$. We define a sequence

$$
y_{0}(\omega)=x_{0}(\omega) \text { and } y_{n}(\omega)=T\left(\omega, y_{n-1}(\omega)\right)=T^{n}\left(\omega, x_{0}(\omega)\right),
$$

for all $\omega \in \Omega$ and integers $n \geqslant 1$. From the conditions (ii) and (iii), we have

$$
y_{0}(\omega)=x_{0} \preccurlyeq T\left(\omega, x_{0}(\omega)\right)=y_{1}(\omega)
$$

implies

$$
y_{1}(\omega)=T\left(\omega, y_{0}(\omega)\right) \preccurlyeq T\left(\omega, y_{1}(\omega)\right)=y_{2}(\omega) .
$$

Inductively, we obtain

$$
y_{0}(\omega) \preccurlyeq y_{1}(\omega) \preccurlyeq y_{2}(\omega) \preccurlyeq \cdots \preccurlyeq y_{n-1}(\omega) \preccurlyeq y_{n}(\omega) \preccurlyeq y_{n+1}(\omega) \preccurlyeq \cdots,
$$

or in the second case

$$
y_{0}(\omega) \succcurlyeq y_{1}(\omega) \succcurlyeq y_{2}(\omega) \succcurlyeq \cdots \succcurlyeq y_{n-1}(\omega) \succcurlyeq y_{n}(\omega) \succcurlyeq y_{n+1}(\omega) \succcurlyeq \cdots
$$

Using triangle rule and asymptotic regularity, we get for $m>n$,

$$
\begin{aligned}
& d\left(y_{n}(\omega), y_{m}(\omega)\right) \\
& \quad \leqslant d\left(y_{n}(\omega), y_{n+1}(\omega)\right)+d\left(y_{n+1}(\omega), y_{m+1}(\omega)\right)+d\left(y_{m+1}(\omega), y_{m}(\omega)\right) \\
& \quad=d\left(y_{n}(\omega), y_{n+1}(\omega)\right)+d\left(T\left(\omega, y_{n}(\omega)\right), T\left(\omega, y_{m}(\omega)\right)\right)+d\left(y_{m+1}(\omega), y_{m}(\omega)\right) \\
& \quad \leqslant(K(\omega)+1) \cdot\left\{d\left(y_{n}(\omega), y_{n+1}(\omega)\right)+d\left(y_{m}(\omega), y_{m+1}(\omega)\right) \rightarrow 0,\right.
\end{aligned}
$$

as $m>n \rightarrow \infty$. So $\left\{y_{n}(\omega)\right\}_{n}$ is a Cauchy sequence for every $\omega \in \Omega$. Since $X$ is a complete space there exists $y_{*}(\omega) \in X, \omega \in \Omega$, such that $y_{*}(\omega)=$ $\lim _{n \rightarrow \infty} y_{n}(\omega)$. Since $y_{0}(\cdot)$ is measurable, then $y_{1}(\cdot)$ is measurable. Hence, by induction, we can easily prove that for each $n \in \mathbb{N}$, the function $\omega \rightarrow y_{n}(\omega)$ is measurable. The mapping $y_{*}: \Omega \rightarrow X$ is the pointwise limit of measurable mappings, so it is measurable.

Now, we show that $y_{*}$ is a random fixed point of $T$, i.e. $y_{*}(\omega)=$ $T\left(\omega, y_{*}(\omega)\right), \omega \in \Omega$. It is clear, for each $\omega \in \Omega$, by asymptotic regularity, we get

$$
\lim _{n \rightarrow \infty} d\left(y_{n}(\omega), y_{n+1}(\omega)\right)=\lim _{n \rightarrow \infty} d\left(T^{n}\left(\omega, x_{0}(\omega)\right), T^{n+1}\left(\omega, x_{0}(\omega)\right)\right)=0,
$$

and because $T(\omega, \cdot)$ is continuous,

$$
d\left(y_{*}(\omega), T\left(\omega, y_{*}(\omega)\right)\right)=\lim _{n \rightarrow \infty} d\left(y_{n}(\omega), T\left(\omega, y_{n}(\omega)\right)\right)=0 .
$$

Thus,

$$
y_{*}(\omega)=T\left(\omega, y_{*}(\omega)\right) \quad \text { for each } \omega \in \Omega
$$


It remains for us to show that $y_{*}$ is the unique random fixed point of $T$. We prove that, if we take any random variable $\bar{x}_{0}: \Omega \rightarrow X$ and we define the sequence

$$
\bar{y}_{0}(\omega)=\bar{x}_{0}(\omega) \quad \text { and } \quad \bar{y}_{n}(\omega)=T\left(\omega, \bar{y}_{n-1}(\omega)\right)=T^{n}\left(\omega, \bar{x}_{0}(\omega)\right)
$$

for all $\omega \in \Omega$ and integers $n \geqslant 1$, we get $\bar{y}_{n}(\omega) \rightarrow y_{*}(\omega)$, as $n \rightarrow \infty$, for every $\omega \in \Omega$, where $y_{*}$ is the random fixed point of $T$ obtained in the previous part of the proof.

If $\bar{x}_{0}(\omega)$ is comparable to $x_{0}(\omega)$ for every $\omega \in \Omega$, it is obvious, since $T\left(\omega, \bar{x}_{0}(\omega)\right)$ is comparable to $T\left(\omega, x_{0}(\omega)\right)$ for every $\omega \in \Omega$, so that $\bar{y}_{n}(\omega)$ is comparable to $y_{n}(\omega)$ for every $\omega \in \Omega$. Hence, by $(\mathrm{v})$ and asymptotic regularity

$$
\begin{aligned}
& d\left(y_{n}(\omega), \bar{y}_{n}(\omega)\right)=d\left(T\left(\omega, y_{n-1}(\omega)\right), T\left(\omega, \bar{y}_{n-1}(\omega)\right)\right) \\
& \quad \leqslant K(\omega) \cdot\left\{d\left(y_{n-1}(\omega), T\left(\omega, y_{n-1}(\omega)\right)\right)+d\left(\bar{y}_{n-1}(\omega), T\left(\omega, \bar{y}_{n-1}(\omega)\right)\right)\right\} \\
& \quad=K(\omega) \cdot\left\{d\left(y_{n-1}(\omega), y_{n}(\omega)\right)+d\left(\bar{y}_{n-1}(\omega), \bar{y}_{n}(\omega)\right)\right\} \rightarrow 0, \omega \in \Omega,
\end{aligned}
$$

as $n \rightarrow \infty$. Therefore

$$
d\left(\bar{y}_{n}(\omega), y_{*}(\omega)\right) \leqslant d\left(\bar{y}_{n}(\omega), y_{n}(\omega)\right)+d\left(y_{n}(\omega), y_{*}(\omega)\right) \rightarrow 0,
$$

as $n \rightarrow \infty$, and $\bar{y}_{n}(\omega) \rightarrow y_{*}(\omega)$, as $n \rightarrow \infty$, for every $\omega \in \Omega$.

On the other hand, for an arbitrary random variable $\bar{x}_{0}: \Omega \rightarrow X$ then, for each $\omega \in \Omega$, there exists $z(\omega) \in X$ that is comparable to $x_{0}(\omega)$ and $\bar{x}_{0}(\omega)$ simultaneously, then if we define the sequence

$$
z_{0}(\omega)=z(\omega) \text { and } z_{n}(\omega)=T\left(\omega, z_{n-1}(\omega)\right)=T^{n}(\omega, z(\omega)),
$$

for all $\omega \in \Omega$ and integers $n \geqslant 1$, then $y_{n}(\omega)$ is comparable to $z_{n}(\omega)$, for every $\omega \in \Omega$, and $\bar{y}_{n}(\omega)$ is comparable to $z_{n}(\omega)$, for every $\omega \in \Omega$. Therefore

$$
\begin{aligned}
& d\left(y_{n}(\omega), \bar{y}_{n}(\omega)\right) \leqslant d\left(y_{n}(\omega), z_{n}(\omega)\right)+d\left(z_{n}(\omega), \bar{y}_{n}(\omega)\right) \\
&=d\left(T\left(\omega, y_{n-1}(\omega)\right), T\left(\omega, z_{n-1}(\omega)\right)\right)+d\left(T\left(\omega, z_{n-1}(\omega)\right), T\left(\omega, \bar{y}_{n-1}(\omega)\right)\right) \\
& \leqslant K(\omega) \cdot\left\{d\left(y_{n-1}(\omega), y_{n}(\omega)\right)+d\left(z_{n-1}(\omega), z_{n}(\omega)\right)\right\} \\
& \quad+K(\omega) \cdot\left\{d\left(z_{n-1}(\omega), z_{n}(\omega)\right)+d\left(\bar{y}_{n-1}(\omega), \bar{y}_{n}(\omega)\right)\right\} \rightarrow 0, \omega \in \Omega,
\end{aligned}
$$

as $n \rightarrow \infty$, which proves that $\bar{y}_{n}(\omega) \rightarrow y_{*}(\omega)$, as $n \rightarrow \infty$, for every $\omega \in \Omega$. This proves the theorem.

Remark 4.12. Theorem 4.11 remains true when condition (v) is replaced by $\left(\mathrm{v}^{\prime}\right)$ there exists functions $M: \Omega \rightarrow[0,1)$ and $K: \Omega \rightarrow[0, \infty)$ such that for each $\omega \in \Omega$,

$$
\begin{aligned}
& d(T(\omega, x), T(\omega, y)) \\
& \quad \leqslant M(\omega) \cdot d(x, y)+K(\omega) \cdot\{d(x, T(\omega, x))+d(y, T(\omega, y))\}
\end{aligned}
$$

for every comparable $x, y \in X$, i.e. $x \preccurlyeq y$ or $y \preccurlyeq x$.

Conclusion. In this part, we presented the extension of Banach's theorem in stochastic situations. 
Open Access. This article is distributed under the terms of the Creative Commons Attribution 4.0 International License (http://creativecommons.org/licenses/by/4. $0 /$ ), which permits unrestricted use, distribution, and reproduction in any medium, provided you give appropriate credit to the original author(s) and the source, provide a link to the Creative Commons license, and indicate if changes were made.

Publisher's Note Springer Nature remains neutral with regard to jurisdictional claims in published maps and institutional affiliations.

\section{References}

[1] Agarwal, R.P., Karapinar, E., O'Regan, D., Rolánd-López-de-Hiero, A.F.: Fixed Point Theory in Metric Type Spaces. Springer International Publishing, Basel (2015)

[2] Ayerbe Toledano, J.M., Domínguez Benavides, T., López Acedo, G.: Measures of Noncompactness in Metric Fixed Point Theory. Birkhäuser, Basel (1997)

[3] Baillon, J.-B., Bruck, R.E., Reich, S.: On the asymptotic behavior of nonexpansive mappings and semigroups in Banach spaces. Houst. J. Math. 4, 1-9 (1978)

[4] Banach, S.: Sur les opérations dans les ensembles abstraits at leur application aux équations intégrales. Fundam. Math. 3, 133-181 (1922)

[5] Berinde, V.: Iterative Approximation of Fixed Points. Springer, Berlin (2007)

[6] Bharucha-Reid, A.T.: Random Integral Equations. Academic Press, New York (1972)

[7] Browder, F.E., Petryshyn, W.V.: The solution by iteration of nonlinear functional equations in Banach spaces. Bull. Am. Math. Soc. 72, 571-576 (1966)

[8] Bruck, R.E., Reich, S.: Nonexpansive projections and resolvents of accretive operators in Banach spaces. Houst. J. Math. 3, 459-470 (1977)

[9] Carl, S., Heikkilä, S.: Fixed Point Theory in Ordered Sets and Applications, from Differential and Integral Equations to Game Theory. Springer, New York (2011)

[10] Chatterjea, S.K.: Fixed-point theorems. C.R. Acad. Bulgare Sci 25, 727-730 (1972)

[11] Connell, E.H.: Properties of fixed point spaces. Proc. Am. Math. Soc. 10, 974979 (1959)

[12] De Blasi, F.S.: Fixed points for Kannan's mappings in Hilbert spaces. Boll. Un. Mat. Ital. (4) 9, 818-823 (1974)

[13] Edelstein, M., O'Brien, R.C.: Nonexpansive mappings, asymptotic regularity and successive approximation. J. Lond. Math. Soc. 17, 547-554 (1978)

[14] Emmanuele, G.: Fixed point theorems in complete metric space. Nonlinear Anal. 5, 287-292 (1981)

[15] Espínola, R., Wiśnicki, A.: The Knaster-Tarski theorem versus monotone nonexpansive mappings. Bull. Polish Acad. Sci. Math. 66, 1-7 (2018)

[16] Goebel, K., Kirk, W.A.: Iteration Processes for Nonexpansive Mappings, Contemporary Mathematics, vol. 21, pp. 115-123. AMS, Providence (1983)

[17] Goebel, K., Kirk, W.A.: Topics in Metric Fixed Point Theory. Cambridge University Press, Cambridge (1990) 
[18] Goebel, K., Reich, S.: Uniform Convexity, Hyperbolic Geometry and Nonexpansive Mappings. Marcel Dekker, New York (1984)

[19] Górnicki, J.: A fixed point theorem for asymptotically regular mapping. Colloq. Math. 64, 55-57 (1993)

[20] Górnicki, J.: Fixed points of asymptotically regular semigroups in Banach spaces. Rend. Circ. Mat. Palermo Ser. II 46, 89-118 (1997)

[21] Górnicki, J.: On the structure of fixed point sets of asymptotically regular mappings in Hilbert spaces. Topol. Methods Nonlinear Anal. 34, 383-389 (2009)

[22] Górnicki, J.: Geometrical coefficients and the structure of the fixed-point set of asymptotically regular mappings. Nonlinear Anal. 74, 1190-1199 (2011)

[23] Górnicki, J.: Structure of the fixed point set of asymptotically regular mappings in uniformly convex Banach spaces. Taiwan. J. Math. 15, 1007-1020 (2011)

[24] Górnicki, J.: Remarks on contractive type mappings. Fixed Point Theory Appl. 2017, 8 (2017). https://doi.org/10.1186/s13663-017-0601-4

[25] Górnicki, J.: Fixed point theorems for Kannan type mappings. J. Fixed Point Theory Appl. 19, 2145-2152 (2017). https://doi.org/10.1007/ s11784-017-0402-8

[26] Granas, A., Dugundji, J.: Fixed Point Theory. Springer, New York (2003)

[27] Hanš, O.: Random fixed point theorems. In: Transactions of the First Prague Conference on Information Theory, Statistical Decision Functions, Random Processes, Liblice near Prague (28-30 Nov. 1956). Publ. House Czechoslovak Acad. Sci., Prague, pp. 105-125 (1957)

[28] Hillam, B.P.: A characterization of the convergence of successive approximations. Am. Math. Mon. 83, 273 (1976)

[29] Himmelberg, C.J.: Measurable relations. Fundam. Math. 87, 53-72 (1975)

[30] Ishikawa, S.: Fixed points nad iteration of nonexpansive mapping in Banach space. Proc. Am. Math. Soc. 59, 65-71 (1976)

[31] Ivanov, A.A.: Fixed points of mappings of metric spaces (Russian). Zapiski Nauch. Sem. Leningrad. Otd. Mat. Inst. Steklova AN SSSR 66, 5-102 (1976)

[32] Kannan, R.: Some results on fixed points. Bull. Calcutta Math. Soc. 60, 71-76 (1968)

[33] Kirk, W.A., Shahzad, N.: Fixed Point Theory in Distance Spaces. Springer International Publishing, Basel (2014)

[34] Lin, P.-K.: A uniformly asymptotically regular mapping without fixed point. Can. Math. Bull. 30, 481-483 (1987)

[35] Nieto, J.J., Ouahab, A., Rodríguez-López, R.: Random fixed point theorems in partially ordered metric spaces. Fixed Point Theory Appl. 2016, 98 (2016). https://doi.org/10.1186/s13663-016-0590-8

[36] Nieto, J.J., Rodríguez-López, R.: Contractive mapping theorems in partially ordered sets and applications to ordinary differential equations. Order 22, 223239 (2005)

[37] Ran, A.C.M., Reurings, M.C.B.: A fixed point theorem in partially ordered sets and some applications to matrix equations. Proc. Am. Math. Soc. 132, 1435-1443 (2003)

[38] Reem, D., Reich, S.: Zone and double zone diagrams in abstract spaces. Colloq. Math. 115, 129-145 (2009) 
[39] Reich, S.: Some remarks concerning contraction mappings. Can. Math. Bull. 14(1), 121-124 (1971)

[40] Reich, S.: A random fixed point theorem for set-valued mappings. Atti Accad. Naz. Lincei 64, 65-66 (1978)

[41] Reich, S., Shafrir, I.: The asymptotic behavior of firmly nonexpansive mappings. Proc. Am. Math. Soc. 101, 246-250 (1987)

[42] Reich, S., Zaslavski, A.J.: Monotone contractive mappings. J. Nonlinear Var. Anal. 1, 391-401 (2017)

[43] Rech, S., Zaslavski, A.J.: Generic well-posedness of the fixed point problem for monotone nonexpansive mappings. In: Bellow, A., Claude, C.C., Zamfirescu, T. (eds.) Mathematics Almost Everywhere, in Memory of Solomon Marcus, pp. 169-179. World Scientific, Singapore (2018)

[44] Smart, D.R.: When does $T^{n+1} x-T^{n} x \rightarrow 0$ imply convergence? Am. Math. Mon. 87, 748-749 (1980)

[45] Špaček, A.: Zufällige Gleichungen. Czechoslov. J. Math. 5(80), 462-466 (1955)

[46] Subrahmanyam, P.V.: Completness and fixed points. Monatsch. Math. 80, 325330 (1975)

[47] Wagner, D.-H.: Survey of mesurable selection theorems. SIAM J. Control Optim. 15, 859-903 (1977)

[48] Wiśnicki, A.: On the structure of fixed-point sets of asymptotically regular semigroups. J. Math. Anal. Appl. 393, 177-184 (2012)

[49] Zamfirescu, T.: Fix point theorems in metric spaces. Arch. Math. (Basel) 23, 292-298 (1972)

Jarosław Górnicki

Department of Mathematics and Applied Physics

Rzeszów University of Technology

P.O. Box 85

35-959 Rzeszow

Poland

e-mail: gornicki@prz.edu.pl 